\title{
CUIDADOS DE LA SALUD: PARADIGMA DEL PERSONAL DE ENFERMEROS EN MÉXICO - LA RECONSTRUCCIÓN DEL CAMINO
}

\author{
Cuidados de saúde: paradigma de enfermeiros no México - \\ a reconstrução da estrada \\ Health care: paradigm of nurses in Mexico - rebuilding the road
}

María de Lourdes García Hernández

Lucila Cárdenas Becerril ${ }^{3}$
Beatriz Arana Gómez

Araceli Monroy Rojas ${ }^{4}$

\section{RESUMEN}

Se hace un recuento histórico-sociológico sobre los antecedentes y las bases filosóficas basada en el rendimiento del personal profesional de enfermería en México, que permiten conocer y reconocer la necesidad apremiante de transitar del paradigma salud-enfermedad al de vida-salud. Esto responde a las necesidades de la población en el ámbito de la salud, en una sociedad donde la atención es a la enfermedad y no a la salud, otorgándose aquélla en un sistema hospitalario y con costos altos. Es una investigación cuya metodología se basa en el enfoque de la sociología de las profesiones, apoyada en entrevistas a profundidad. La población de estudio fueron 10 enfermeras de un hospital público de segundo nivel de atención a la salud. Los resultados indican que mientras la atención de enfermería se otorgue en un área hospitalaria, el paradigma vida-salud tardará en instalarse en el ser y hacer de las enfermeras.

Palabras clave: Enfermería. Atención de enfermería. Prestación de Atención de Salud

\section{Resumo}

Faz-se uma pesquisa histórico-sociológica dos antecedentes e das bases filosóficas que fundamentam o desempenho do pessoal profissional de enfermagem no México, os quais permitam conhecer e reconhecer a necessidade urgente de transitar do paradigma saúde-enfermidade ao de vida-saúde. Isso responde às necessidades da população no âmbito da saúde, em uma sociedade na qual a atenção radica na doença, e não na saúde, sendo esta outorgada em um sistema hospitalar e com altos custos. É uma pesquisa cuja metodologia se baseia no enfoque da sociologia das profissões, apoiada em amplas entrevistas. A população de estudo se conformou de 10 enfermeiros de um hospital público de segundo nível de atenção à saúde. Os resultados indicam que enquanto a atenção de enfermagem se outorgar em uma área hospitalar, o paradigma vida-saúde irá demorar em se instalar no ser e fazer dos enfermeiros.

Palavras chave: Enfermagem. Cuidados de Enfermagem. Assistência à Saúde.

\begin{abstract}
This is a historical-sociological research about the background and philosophical foundations that bases the performance of the professional nursing staff in Mexico, to know and recognize the urgent needs to move from the paradigm of health and disease to life-health. This responds to the needs of the population in the area of health in a society where the attention is to disease rather than to health, and being given at a hospital system and at high costs. This is a research whose methodology was based on the sociology approach of the professions, supported by in-depth interviews. The study population was a group of 10 nurses from a second-level public health care hospital. The results indicate that while nursing care is given at a hospital area, the paradigm life-health will take time to be settled in and to be done by the nurses.
\end{abstract}

Keywords: Nursing. Nursing Care. Delivery of Health Care

${ }^{1}$ Docente de tiempo completo de la Facultad de Enfermería y Obstetricia de la Universidad Autónoma del Estado de México. Alumna del cuarto semestre del Doctorado en Ciencias de la Salud con Maestría en Enfermería con énfasis en Administración en los Servicios de Enfermería, integrante del Cuerpo Académico "Ejercicio de Enfermería". Investigadora adjunta. México. E-mail: luygaba@yahoo.com.mx, ²Docente en la Facultad de Enfermería y Obstetricia de la Universidad Autónoma del Estado de México. Integrante del Cuerpo Tutoral del Doctorado en Ciencias de la Salud y del Cuerpo Académico "Ejercicio de Enfermería", Doctora en Enfermería por la Universidad de Sao Paulo, Brasil. Responsable del proyecto Calidad del cuidado de la salud. México. E-mail betya18@yahoo.com.mx, ${ }^{4}$ Docente de Tiempo Completo la Facultad de Enfermería y Obstetricia de la Universidad Autónoma del Estado de México. Integrante del Sistema Nacional de Investigadores Nivel 1. Responsable del proyecto Calidad del cuidado de la salud. Doctora en Educación por la Universidad Autónoma de Tlaxcala. México. E-mail: lucycabe62@yahoo.com, ${ }^{4}$ Docente de la Universidad Autónoma Metropolitana Xochimilco. Integrante del Sistema Nacional de Investigadores Nivel 1. Participa como tutora externa de la alumna del Doctorado en Ciencias de la Salud. Doctora en Educación por la Universidad de Tlaxcala. México. E-mail: aramonroy@yahoo.com. 


\section{INTRODUCCIÓN}

El personal profesional de Enfermería debe tener claridad sobre su objeto de estudio y trabajo, es decir, de la importancia que tiene que sus objetivos, metas e intervenciones se enfoquen a fomentar y preservar la salud de la persona, de la familia y de la sociedad. Esto implica, entre otras concepciones, ubicar a la salud y a su naturaleza, como el paradigma eje que guíe su ejercicio profesional. Este artículo tiene por objetivo hacer un recuento histórico-sociológico sobre los antecedentes y las bases filosóficas que subyacen en el personal profesional de enfermería en México, que permitan conocer y reconocer la necesidad apremiante de transitar del paradigma salud-enfermedad al de vida-salud. Ello responde a las necesidades de la población en el ámbito de la salud, en una sociedad donde la atención es a la enfermedad y no a la salud, otorgándose aquélla en un sistema hospitalario y con costos altos.

Esto nos lleva a conocer los límites y alcances del cuidado que otorga el personal de enfermería en un sistema de salud caracterizado, todavía, por una atención mayormente biologicista, ahistórica e individual, hospitalaria, curativa y por una fragmentación en la atención del equipo interdisciplinario de salud, cuya profesión hegemónica sigue siendo la medicina.

La salud es un término que puede adoptar significados diversos dependiendo del uso y contexto en que se utiliza. Desde el punto de vista filosófico, la salud se define como un hábito psico-orgánico al servicio de la vida y de la libertad de la persona, que consiste tanto en la posesión de una normalidad como en la capacidad física para realizar los proyectos vitales de la persona'. La Organización Mundial de la Salud (OMS), la define como el estado de perfecto bienestar físico, psíquico y social, y no sólo la ausencia de lesión o enfermedad. En esta definición se integran dos elementos: la integridad física y el bienestar del sujeto. En la antigüedad no se tenía dificultad para alcanzar una idea clara de lo que era salud, porque como se hablaba en latín, la sola palabra salus daba la idea de su significado. Salusy salvatio, significaban estar en condiciones de poder superar un obstáculo. De estas palabras latinas se derivan sus equivalentes castellanas: salud y salvación. El término castellano salvarse incluye el significado original de superar una dificultad. Sin embargo, el término salud no se entiende actualmente como ligado a dicho significado².

La salud incluye cier to grado de bienestar físico y es una parte altamente significativa de la vida, es decir, es uno de los medios necesarios para seguir viviendo. La ciencia moderna ha ignorado que el hombre se relaciona en un sistema de derechos y obligaciones para cuidar su salud y apartarse de la enfermedad. El derecho a la salud no es más que el derecho a la vida. Por tanto, estar sano significa estar en sincronía con uno mismo y con otras formas de vida que nos rodean. De esta manera, la salud y la vida pueden mirarse como la misma cosa, porque ambas se implican`.
Por su parte, la vida no es exclusivamente biológica, es una realidad compleja donde se conjugan los aspectos biológicos, psicológicos, sociales y espirituales. Por tanto, seguir viviendo, en el caso del hombre, no sólo es mantener la vida biológica y reproducirse, sino también es importante poder actuar con su voluntad, llevando a cabo sus actividades en la vida diaria. Este paradigma tiene consecuencias sobre la práctica de enfermería, al determinar que el fin que debe perseguirse es cuidar la salud del paciente para asegurar el bienestar.

Considerando a la salud como un bienestar integral y concediendo no sólo importancia a las determinantes biológicas y genéticas del ser humano, sino también a las interacciones del medio ambiente que propician los comportamientos de adaptación manifestados a través del paradigma vida-salud, la atención a la salud en nuestra época requiere de un ejercicio interprofesional, donde cada profesionista posea conocimiento científico y actitudes de compromiso profesional, ético y social para enfrentar y resolver en forma integral los problemas que impiden la detección oportuna de riesgos, el autocuidado a la salud, la prevención de enfermedades, así como el diagnóstico y reducción de enfermedades y sus consecuencias individuales y sociales.

En este sentido, cuidar de la salud se convierte en un vínculo de relaciones en el que los profesionales de Enfermería ofertan sus servicios a la demanda de cuidados que ofrecen los diversos sistemas de salud.

Bajo esa óptica, el hacer de enfermería se ve implicada en una dinámica de un sistema de atención institucionalizado, en donde, las instituciones de salud no han afrontado el cambio de escenario para una práctica interprofesional que favorezca el crecimiento de la enfermería. Paralelamente, las instituciones educativas no han logrado ponderar en qué medida deben prepararse sus egresados para contender con un mercado de trabajo que sólo exige rendimiento laboral más que productividad profesional. También es preocupante la subutilización que se hace de la competencia de enfermería para trabajar un modelo de atención a la salud en el que predomine la labor educativa para lograr un cambio en la población, que permita la adquisición de una nueva cultura en salud, en la que destaque la importancia del cuidado del individuo y de su medio ambiente como medidas preventivas de riesgos y de enfermedades ${ }^{3}$.

Con base en lo señalado con antelación, el tránsito del ser y hacer de las enfermeras desde el paradigma salud-enfermedad al de vida-salud, se torna lento y difícil, toda vez que los antecedentes del surgimiento de la enfermería moderna, las condiciones que establece el sistema de salud y, en general, las influencias económicas, políticas, culturales, educativas y laborales, son factores decisivos para el cambio esperado en los albores del siglo XXI. 


\section{REVISION DE LITERATURA: EL CAMINO RECORRIDO}

A nivel mundial, se considera que el siglo XIX vio nacer a la Enfermería moderna ${ }^{a}$ con el trabajo de Florence Nightingale, quien aportó las bases técnico-administrativas de Enfermería, creando un modelo teórico de atención $n^{4}$. En este marco surge la Enfermería en México, ${ }^{b}$ producto de la necesidad de un personaje que cuidara al enfermo y al desvalido; una vez que el médico diagnosticara y tratara al paciente. De hecho, son los propios médicos quienes se percatan de dicha necesidad y proponen e impulsan la creación de la primera escuela de Enfermería en 1907. Como campo de conocimiento, es ahí donde se delimita, puesto que a la medicina le queda claro que su ámbito de competencia no es el cuidar, sin embargo, por las características del surgimiento de la enfermería, históricamente la medicina establece las bases de la Enfermería en los primeros 20 años del siglo XX.

Este acto generó algunas características que se han matizado a través de casi una centuria. En primer término, las actividades de las enfermeras conformaron un modelo de atención empírico-práctico, es decir, sus acciones se basaban fundamentalmente en el método del aprendiz, se daba respuesta al cómo y, en pocas ocasiones, al por qué de dicha actividad; aunado a ello, lo que aprendieron formaba parte de la medicina biologicista imperante: lograr primacía del cuerpo por sobre lo psicológico o espiritual.

Principalmente durante las prácticas en el hospital, cuando las enfermeras recibían el conocimiento de otras a través del método del aprendiz; la estudiante de enfermería era la aprendiz y la enfermera graduada y con experiencia era la maestra; esta forma de enseñar aún se sigue utilizando en la actualidad. La atención al paciente y a la parturienta no se aprendía en libros ni tampoco de los maestros médicos, sino de las relaciones amistosas que se establecieran con enfermeras y obstetras del hospital. Nadie explicaba ni decía nada, nadie hablaba de fundamentos teóricos que ahora se enseñan. Los cambios han sufrido los programas de enseñanza de la enfermería, se sobreentiende que fueron para mejorar la calidad de la atención, pero pareciera que la ventaja se ha reducido a los aspectos técnicos. Lo eminentemente humano, el trato de persona a persona, ha ido en detrimento, fenómeno que está vinculado con toda la problemática de los efectos deshumanizantes de la revolución científico-tecnológica ${ }^{5}$.

En lo que respecta a la atención del cuerpo, de lo biológico, lo individual y segmentado, tiene sus orígenes a fines del siglo XVIII, cuando los hospitales se convierten en centros de atención a los enfermos ${ }^{6}$. Es ahí donde se determina que la percepción médica no debe dirigirse ni a las series, ni a los grupos; debe estructurarse como una mirada a través de una lupa, que aplicada a las diferentes partes de un objeto, hace aún notar en él otras partes que no se percibían sin ella y emprender el infinito trabajo del conocimiento del individuo ${ }^{7}$.
Las enfermeras mexicanas transitan los primeros 50 años del siglo pasado, por un desarrollo que comienza a cobrar matices, tanto en conocimientos, prácticas e ideas; ocurren algunos cambios que permiten avizorar mejorías en la profesión. Por principio de cuentas, las escuelas de enfermería dejan de depender de los hospitales, se formalizan los planes y programas de estudio, se solicita la secundaria para ingresar a la carrera, se privilegia la formación teórico-práctica; los dirigentes son médicos, pero se incorporan a la docencia enfermeras; se institucionaliza la Enfermería, incorporándose al sistema universitario, entre otros cambios educativos. Los siguientes 30 años (1950-1980) las enfermeras cobraron relevancia en el sistema de salud, demostrando su conocimiento y pericia, tanto en la salud pública, como en las diferentes especialidades hospitalarias; la formación y actualización fue uno de los ejes que las guió por el camino del reconocimiento y prestigio", ello se traducía en enfermeras que, aún cuando sus estudios eran técnicos, contaban con dos o tres especialidades. En términos amplios, este período se caracterizó por privilegiar la teoría sobre la práctica; enseñando el Proceso Atención de Enfermería (PAE). Las enfermeras mexicanas enfocaron su atención al paciente, casi siempre hospitalizado, y a responder a las expectativas biologicistas de especialización; apoyadas por el modelo curativo prevaleciente, sin considerar algunos aspectos de tipo social, como las valoraciones que se han hecho de las distintas profesiones, incluida Enfermería; lo que genera un estatus y un prestigio, que además se traduce en un ingreso económico.

A partir de la década de 1980 y hasta nuestros días, las enfermeras se han cuestionado su ser, hacer profesional, la preocupación latente por los fundamentos teóricos y científicos de la profesión, enmarcada principalmente por el abordaje de las Teorías y Modelos en Enfermería y por la definición y consenso del cuidado como objeto de estudio8.

\section{Paradigmas de enfermería en la actualidad}

Innegablemente, los saberes y las prácticas de la profesión de Enfermería tienen una fuerte herencia empírica. Las mujeresenfermeras no sólo aportan su sabiduría; misma que desciende de mujeres cuidadoras, sino que imprimen en su actuar un valor agregado, el enfoque humanístico, la sensibilidad empática de buscar alivio, consuelo y armonía a uno de los valores más preciados de la humanidad: la salud, preservando con ello una vida digna o una muerte asistida desde el punto de vista técnico y humanístico.

En este sentido ontológico, ser mujer-enfermera es una ventaja demostrada, a través de todos los tiempos, para cuidar, en el sentido más amplio; a todo ser humano que se encuentre en desequilibrio biopsicosocial, espiritual y cultural; es decir, enfermo. Los conocimientos formales que adquirieron las enfermeras a razón de la creación de la primera escuela de Enfermería en México, tuvieron dos características. En primer término, el enfoque teórico fue eminentemente biologicista; en 
segundo, los médicos enseñaban lo que consideraban que una enfermera debía saber.

En la actualidad las enfermeras buscan transformar la práctica profesional, mediante la transmisión de saberes que la propia profesión considera de vanguardia, con ellos nos referimos a la enseñanza del Proceso de Enfermería y a las Teorías y Modelos. Ambos enfoques buscan establecer y delimitar el trabajo de Enfermería, es decir el cuerpo de saberes. Sin embargo, las enfermeras que se encuentran insertas en el mercado laboral, en su mayoría en hospitales, no utilizan un modelo de atención que por lo menos dé cuenta de un trabajo sistematizado. Por otro lado, las características en la enseñanza y posesión de los saberes y prácticas hace que puedan distinguirse dos mundos en la Enfermería mexicana; el académico y el hospitalario, puesto que mientras en el ámbito educativo se propugna por mayores niveles académicos. En el área hospitalaria se observan políticas de atención que privilegian la productividad y la calidad.

En lo cotidiano, la vinculación docencia-servicio continua siendo el desiderátum de la profesión desde mediados del siglo XX, en la medida en que el acercamiento y la realimentación de ambos procesos -el asistencial y el educativo-, se ven influenciados por los sistemas laborales disímiles, lo que ha dificultado que las enfermeras se muestren solidarias con la profesión, en términos gremiales y de identidad; y no solamente identificadas con la institución empleadora. De ahí que la formación y los saberes de enfermería deberán conciliarse con las formas de trabajo en el mercado laboral del personal de enfermería ${ }^{3}$.

\section{El cuidado de la salud, paradigma de enfermería en los albores del siglo XXI}

Como se ha señalado con antelación, las enfermeras desarrollan una serie de actividades encaminadas a constituirse como disciplina científica. En ese contexto, buscan sistematizar su conocimiento empírico para ampliar la concepción del Proceso de Enfermería hacia un diagnóstico en Enfermería; abordando el paradigma vida-salud de manera distinta al acto médico, lo que significa trabajar en la conformación de un pensamiento profesional propio, ampliando la manera de entenderse a sí misma y al objeto de su actividad profesional; considerando sus labores más allá de la simple ocupación, elevándolas para alcanzar los niveles de profesión; ${ }^{8}$ es decir, la profesión se encuentra en fase preparadigmática, en la constitución del andamiaje que le dé sustento. Tal condición se aplica tanto al ámbito académico como al de servicio, mediante el análisis y recreación de propuestas filosóficas, teóricas y metodológicas para la construcción del cuerpo de saberes de enfermería. Lo anterior permitirá fortalecer los fundamentos de la disciplina y, por ende, perfeccionar la práctica cotidiana a través de la descripción, explicación, predicción y control de los fenómenos del cuidado a la salud, al mismo tiempo, hará posible la autonomía en la formación de recursos humanos competentes para el desempeño en las diferentes áreas asistenciales que requiere la sociedad y, simultáneamente, fortalecerá el proceso de profesionalización de enfermería en el país 9 .

En sentido epistémico, hay acuerdo sobre los cuatro conceptos centrales que soportan el desarrollo de la enfermería en lo disciplinar y en lo profesional. Estos cuatro conceptos se han identificado como hombre (persona: paciente o usuario, y enfermera), contexto (ambiente: del paciente y de la enfermera), salud (vida) y cuidado (acción, intervención terapéutica) de enfermería. Las estructuras conceptuales son de vital importancia en este momento en la evolución de la ciencia de enfermería y ayudan asía esclarecer las diferencias entre la enfermería y las demás profesiones dedicadas a la salud.

Existe también consenso en que el objeto de estudio de la profesión de enfermería es el cuidado del ser humano, desde la concepción hasta la muerte, con un enfoque de vida-saludy no de salud-enfermedad. Aunque el acto de cuidar es propio de la naturaleza humana, la enfermería se apropia y asume el cuidado como esencia de su profesión. Desde su marco conceptual, las enfermeras conciben al cuidar como un acto de vida, en el sentido que implica una serie de actitudes y actividades dirigidas a conservar y mantener la vida. Es un acto individual de quien da el cuidado y de reciprocidad con quien requiere ayuda, para asumir sus necesidades vitales a lo largo del proceso de desarrollo humano ${ }^{10}$.

La función primordial de la enfermera consiste en mantener la vida, asegurando la satisfacción de un conjunto de necesidades indispensables para la misma, pero que son diversas en sus manifestaciones. La valoración de los diferentes factores biológicos, psicológicos y sociales que llevaron a la persona a tal situación de alteración de su salud, es la esencia del cuidado; mismo que se puede otorgar por la enfermera profesional, de manera autónoma, en la medida en que está capacitada para tomar decisiones en su campo disciplinario y/o en forma interdependiente, porque la atención de la salud es tan amplia $y$ tan compleja que requiere del trabajo interprofesional, tanto en el ámbito hospitalario como en la comunidad. El holismo en enfermería, hace alusión al carácter global e integrador del cuidado que se otorga a la persona, la cual también posee en su esencia su propio individualismo ${ }^{11}$.

El cuidado es una forma de expresión, de relación con el otro ser y con el mundo, como una forma de vivir plenamente. Cuidar significa comportamientos y acciones que envuelven conocimientos, valores, habilidades y actitudes, emprendidas en el sentido de favorecer las potencialidades de las personas para mantener o mejorar la condición humana en el proceso de vivir y morir. Cuidar implica comprender y ser comprendido, buscando el crecimiento y desarrollo de la persona, del ser ahí en el mundo. El cuidado es "como una forma ética y estética de vivir, que se inicia por el amor a la naturaleza y pasa por la apreciación de lo bello. Consiste en el respeto a la dignidad humana, en la sensibilidad para con el sufrimiento y en la ayuda 
para superarlo, para enfrentarlo y para aceptar lo inevitable. Ese proceso incluye crecimiento ${ }^{12}$.

Los significados más comunes del término cuidar le dan tres sentidos: atención o interés, responsabilidad o cubrir necesidades y consideración o afecto. Madeleine Leininger clasifica el cuidado en tres categorías: Profesional, científico y humano. El primero encarna las metas cognoscitivas intencionadas, los procedimientos y los actos de los profesionales; expresa actitudes e intereses hacia los demás con fines de ayuda para encontrar las necesidades obvias 0 previstas, buscar el bienestar y aliviar los trastornos. El cuidado profesionalse identifica con la prestación de ayuda a los demás mediante actos basados en el conocimiento ensayado y verificado. El cuidado humano está caracterizado por el acto creativo, caritativo, intuitivo o cognoscitivo de ayuda. Se basa en acciones derivadas de los sentimientos y en actos empíricos, filosóficos, fenomenológicos, objetivos y subjetivos de asistencia a otros ${ }^{13}$.

El cuidado de enfermería el ser humano adquiere mayor importancia que la enfermedad. Se trascienden las técnicas y procedimientos, los cuales constituyen sólo parte y medio para lograr la atención integral. Desde el sentido ontológico, el cuidado es una manera de ser, mirar, pensar, compartir y participar con el otro; es describir la experiencia que permite al mismo tiempo el conocimiento propio. Cuidar es una función social y cultural, una ciencia social y humana que demanda preparación, conocimientos y experiencia. Para el cuidado de la persona el profesional de enfermería asume los papeles de proveedor de cuidados, educador para la salud e investigador ${ }^{9} 3$ 5. La enfermería reconoce y conjuga la multidimensionalidad de los factores que influyen en el cuidado profesional de enfermería; las ciencias biológicas, las ciencias sociales y del comportamiento se enlazan para dotarla de elementos sólidos que la ayudan a concebir al ser humano en su dimensión holística ${ }^{14}$.

A casi 30 años de brindar atención y cuidado de enfermería bajo un proceso sistemático y considerar que existe una diferencia significativa entre el diagnóstico y tratamiento que lleva a cabo el médico, y la valoración, el diagnóstico, la intervención y la evaluación que hace el personal de enfermería sobre el cuidado, mediante su método específico, ahora solamente llamado Proceso de Enfermería, puede aseverarse que el cuidado que otorga la enfermera profesional en el área hospitalaria es favorecido por conocimientos innovadores, por la aplicación de la tecnología, por la organización estructural y funcional de la profesión de enfermería en el área hospitalaria y por el acendrado sentido humanístico, característico de las enfermeras.

\section{METODOLOGÍA}

El presente trabajo se deriva del proyecto de investigación, Fundamentación epistemológica del cuidado de la Salud, como objeto de estudio de la enfermera profesional. En un sentido teórico-metodológico, el abordaje se hace desde el enfoque propuesto por la sociología de las profesiones, combinado con una metodología cualitativa basada en entrevistas. Se trata de un estudio exploratorio y, por tanto, de diagnóstico, realizado en un hospital público de segundo nivel de atención del Instituto de Salud del Estado de México (ISEM). La población de estudio fueron 10 enfermeras que se encontraban laborando en los diferentes servicios del hospital en el mes de mayo de 2008. La selección fue de manera intencionada. Los requisitos de inclusión fueron: contar con más de un año de experiencia en su campo laboral, poseer estudios mínimos de licenciatura y aceptar ser entrevistado. Los datos se recolectaron mediante una guía de entrevista semi-estructurada. Se transcribieron de manera literal, posteriormente se empleó el método de análisis de contenido y se construyeron tres núcleos temáticos: $\mathrm{El}$ cuidado de la enfermera profesional, la práctica profesional y su situación académica.

\section{Presentación y discusión de resultados}

Los resultados obtenidos sobre los límites y alcances del cuidado por las enfermeras fueron los siguientes: Las diez enfermeras contaban con una antigüedad laboral promedio de 8 años, con un rango de 18 , toda vez que la que tenía mayor antigüedad era de 20 años y la de menor dos años. La edad de las enfermeras oscila entre los 27 y 43 años, con un promedio de 33 . El $60 \%$ de los participantes tenían nivel de licenciatura, un $20 \%$ especialidad, este mismo porcentaje aplicó en los que tienen estudios de maestría. Los datos de las respuestas se dan a conocer en tres núcleos que a continuación se describen:

\section{La situación del cuidado profesional de la salud}

El cuidado profesional de la salud se define como las acciones que implican un sentimiento emocional o de bienestar de otra persona que van impregnadas del componente científico, esto es, aquellos saberes y acciones de reconocida eficiencia utilizados en la asistencia a individuos y comunidades ${ }^{15}$. En consecuencia, el cuidado profesional que da la enfermera es un acto de vida y al mismo tiempo es recíproco con la persona que desea recobrar su salud. Esto queda evidenciado con las siguientes palabras:

[...] Cuidar la salud son acciones que se realizan
desde el inicio de la vida, todas las enfermeras con
estudios universitarios están capacitadas para dar
cuidados profesionales. Para llevarlo a cabo el
cuidado es necesario que el paciente también
participe, en algunas ocasiones se le solicita al
familiar que lo visita que nos ayude en algunas
intervenciones, que son de tipo técnico. [...](E2).

La apreciación que tienen las enfermeras sobre qué es el cuidado de la salud, permite evidenciar que éste es un acto que se debe realizar durante toda la vida, bajo el acompañamiento de la enfermera como parte de un equipo de salud, con el apoyo de la familia. Como toda actividad humana, 
los cuidados tienen una dimensión ética y moral, que se fundamentan en los principios y valores, que permiten analizar las situaciones para decidir qué es lo correcto. Se da dentro de una interacción dialéctica, que se construye a través de la confianza y progresa con el respeto y el afecto; en dicha interacción se tienen en cuenta los valores de las personas involucradas ${ }^{16}$. Una enfermera cuida la salud cuando reconoce a la persona que requiere de cuidados. Éste es un fenómeno universal, pero los métodos y técnicas con los que se desarrollan varían culturalmente.

[...]Cuido enfermos, siempre en estado crítico, a las familias de los enfermos cuando se en interesan cuidar a su paciente, cuando están en espera de que se agrave, ya que en algunas ocasiones después de que el paciente se recupera, el familiar que esta como cuidador también enferma [...](E7).

[...] Yo cuido pacientes y casi siempre trato de darles cuidados generales, cuando están más graves se tienen que dar cuidados más específicos y con un método y técnica más riguroso. Aunque en algunas ocasiones tengo muchos, por lo regular siempre estoy sola en el servicio, aún así, trato de estar más en contacto con el paciente, a través de la comunicación para conocer sus necesidades más apremiantes $[\ldots](E 8)$.

Las respuestas que dan las enfermeras sobre a quién y cómo se cuida, dejan al descubierto que la enfermera mayormente emplea el paradigma salud-enfermedad, esto es, cuida a enfermos para que recobren su salud. En su mayoría son pacientes crónicos o graves, en quienes tienen que establecer métodos y técnicas de atención de enfermería, de manera sistemática y fundamentada. Algo particularmente importante, es que refieren enseñar a los familiares el cuidado que se le debe proporcionar al enfermo y las formas de cómo debe cuidarse el propio familiar. Por otra parte, al valorar al paciente, la enfermera considera el tiempo que requiere dedicarle a cada paciente, considerando, la gravedad de la enfermedad. En este sentido, las enfermeras tienen el papel fundamental de crear y promover ambientes de cuidado. En donde la enfermera establezca la interconexión que existe entre la salud-cuidado y curación ${ }^{17}$. Desde este punto de vista la enfermera habla sobre el cuidado de la salud, relacionando la enfermedad-salud.

[...]Todos los que trabajamos en el área de la salud cuidamos, pero cada quien tiene sus formas y métodos. A las enfermeras nos toca cuidar que no se agrave el paciente y recobre la salud, además estamos valorando constantemente sus necesidades. Somos los profesionistas que nos coordinamos de una manera directa con el equipo médico, la familia y el personal paramédico, con el fin de proporcionar un cuidado integral al paciente $[. .].(E 3)$.

[...] Las enfermeras no cuidamos de manera aislada lo hacemos de manera multidisciplinaria. Aunque tomamos en cuenta que cuidar al paciente es una función primordial de nosotros como enfermeras $[\ldots](E 6)$.

Respecto a quienes cuidan la salud, se puede mencionar que cuidar, en primer lugar, es una tarea con una dimensión social que está determinada por la interacción con otros profesionales en el ámbito hospitalario, con los pacientes, y con los familiares de éste. En segundo lugar, es una actividad dirigida al ser humano, que es finito, lábil y sensible, y que requiere, en mayor o menor medida, de una atención solícita de otro. Cuidar que el paciente mantenga y recobre la salud es el objetivo de enfermería, por ser el profesional que está en contacto directo con él en todo momento. Para ello, enfermería tiene un cuerpo propio de saberes, que se manifiesta en los cuidados profesionales que otorga ${ }^{18}$. Sobre ello, las entrevistadas opinan lo siguiente:

[...] Es necesario cuidar a las personas para que se curen y recobren la salud. Aunque los aparatos electromédicos y medicamentos nuevos, ayudan a atender al paciente, La falta de cuidados por parte del paciente hace, que en algunos casos, su padecimiento se compliquen por contar con alguna enfermedad crónica como la diabetes, hipertensión o los que se intentan suicidar - que en los últimos meses se han incrementado - aunque también hay otros trastornos, los cuidados que la enfermera proporciona en su mayoría se relacionan a este tipo de pacientes $[\ldots]($ E1).

[...]Cuidamos a las personas para que estén sanas y retornen pronto a su hogar, en algunas ocasiones se puede ver que el paciente se angustia mucho, por que extraña su ambiente. En otros casos, se da de alta al paciente yno desea irse porque le preocupa no tener quien lo cuide y prefiere estar en el hospital. $[\ldots](E 10)$. 
Desde esta óptica las enfermeras cuidan para que las personas estén sanas y se integren a su ambiente lo más pronto posible. Se apoyan en el uso de la tecnología para dar un cuidado más eficiente y evitar complicaciones, ya que tal condición, exige que el paciente permanezca más días en el hospital y su salud se puede deteriorar. A partir de lo que han expresado las enfermeras entrevistadas, creemos que para ellas cuidar es un acto que implica una relación de social con el paciente, con su familia y con el equipo de salud. Resulta relevante la importancia que le dan al apoyo de los familiares para que participen en el cuidado del enfermo.

\section{Actividades que realizan las enfermeras en la práctica profesional}

Como ya ha sido señalado, el personal profesional de enfermería realiza acciones en torno al cuidado, lo que la hace única y diferente en el concier to de las profesiones de la salud. En ese sentido, las enfermeras encuestadas señalan:

[...] Mis actividades se dividen en: dar un cuidado integral al paciente, en algunas ocasiones es necesario recibir material y equipo, revisar que a los pacientes se le hayan realizado los cuidados de la guardia anterior, organizar las actividades que corresponden en el servicio [...](E5).

\section{[...] Lo que hago va en función del tipo de paciente} que tengo que atender y el servicio en el que me asignen. Aunque realmente, casi todo lo que realizó en el hospital está encaminado a atender al paciente, por ejemplo: las que están relacionadas con las intervenciones, otras con la administración -manejo de registros- y también he realizado funciones docentes, participando en sesiones clínicas $[\ldots]($ E4).

\section{[...] Las actividades van desde las técnicas hasta las administrativas y de docencia; todas estas se unifican para atender a un paciente. Aunque en los programas se esta revisando la participación de las enfermeras en la investigación, esta última se me hace muy difícil [...] (E 6).}

En nuestros días, el cuidado de enfermería se concibe como un acto eminentemente humano, caracterizado por el respeto, la confianza y la intimidad, que se da a partir de un juicio de valor para identificar las necesidades y decidir un plan de acción ejecutado, conjuntamente con el paciente y la familia, en el que están en juego el conocimiento, la técnica y la actitud.
[...] lo que realizo con más frecuencia es: recibir pacientes, procedimientos de rutina como: control de soluciones, la higiene del paciente, administro medicamentos. Hay algunas funciones que ya no realizamos, como: instalación de sondas, preparación de alimentación parenteral, e instrumentar en algunas cirugías, lo realizan otros profesionistas [...](E9).

Puede observarse que la práctica profesional de la enfermera se enfrenta a una forma de realizar sus funciones de manera cotidiana, en donde se inserta una sociedad cambiante y con una rápida evolución tecnológica. El $57.1 \%$ de las enfermeras realizan actividades que se refieren a aspectos técnicos que se basan en las características de la atención del sistema de salud y la falta de claridad en los aspectos administrativos, aunque también existen enfermeras poco comprometidas con la profesión que no documentan y sistematizan su trabajo3. De ahí que es necesario revisar, reorganizar y planear sus funciones desde la perspectiva del cuidado del paciente.

\section{Situación académica de la enfermera.}

En la formación de las enfermeras, la educación debe plantear el desarrollo de procesos cognitivos, que les permita responder no sólo para brindar cuidado al paciente y a su familia, sino también para apropiarse de la forma de pensar y actuar en el cuidado, actitud que les apoyará a desarrollar aptitudes que son primordiales para el desarrollo de la profesión.

\section{[...] En el hospital se cuenta con 480 enfermeras, en su mayoría contamos con licenciadas y especialistas, en una minoría, enfermeras con estudios de maestría. Durante todo el año a las enfermeras se les proporcionan sesiones clínicas para reforzar su práctica, éstas están relacionadas con intervenciones de enfermería. Algunas veces solicitan permisos para estudiar y sólo se les autoriza cambiar su turno, ya que, la institución no les permite dejar de trabajar para lograr estudios de otro nivel académico. Es el caso para todos los que laboramos en esta institución [...] (E3).}

Se observa que la formación de las enfermeras se ha incrementado en los últimos veinte años, lo que da a la disciplina una orientación sobre su práctica. Sin embargo, la naturaleza de sus servicios y los alcances de su de responsabilidad, requieren una clara definición de su participación, parte fundamental de la enfermería radica en el crecimiento de su formación y de las teorías que dan sustento al conocimiento en enfermería, pero más que todo, que permitan generar una práctica fundamentada y autónoma. 
[...] Estudié la carrera de licenciado en enfermería y tengo una especialidad postécnica. Esta última la realicé para atender a los pacientes en el área de quemados. Antes no te exigían tantos estudios para trabajar, ahora las nuevas enfermeras deben de contar con título de Licenciatura [... ] (6).

Existe congruencia entre lo señalado por las entrevistadas y la visión que la profesión de enfermería tiene sobre la importancia de educación permanente, hoy llamada educación para la vida. Aunque cabe reconocer que todavía encontramos enfermeras que solamente han tratado de estudiar con el fin de lograr un escalafón en su trayectoria laboral:

[...] yo solo estudié la licenciatura para encontrar un mejor trabajo.. Aunque he tenido cosas buenas, porque desde que estudié trabajo como supervisora, por tanto, no estoy mucho en contacto con los pacientes, sólo cuando es necesario les brindo cuidados [...](2).

Con este ejemplo se puede ver que algunas enfermeras al estudiar mejoran sus condiciones laborales, eso no está mal, sin embargo, es necesario que la enfermera que logre estudios profesionales visualice la importancia de continuar sus estudios en el área, para cuidar con una actitud de compromiso, responsabilidad, presencia y lo más importante que se motive en dirigir sus esfuerzos hacia el cuidado del paciente, de manera eficaz y oportuna.

\section{El camino por recorrer}

El conocimiento especializado que requiere enfermería debe ser único en su estructura, en su organización, de manera que se pueda reclamar propiedad legítima sobre él. No es suficiente que sea conocido sólo por las enfermeras, es indispensable que otros profesionales y el público le den el crédito y el valor. El conocimiento es importante para la enfermería ya que es una disciplina científica y su proceso se basa en la lógica y en el método científico. Como la enfermería ha participado activamente en el desarrollo de una teoría propia durante los últimos veinte años, resulta evidente que está comprometida con sus propias creencias respecto a su existencia profesional. La integración de una filosofía dentro del Proceso de Enfermería y la suposición de que la filosofía de una persona determina sus actos, respaldan la necesidad que tienen los profesionales de enfermería de elaborar y reflexionar sobre su propia filosofía y práctica ${ }^{19}$. La propia Virginia Henderson comentó al respecto que el desafío en enfermería es la contrastación empírica -requisito imprescindible en toda teoría-. "Todo aquel que haya escrito sobre la teoría de enfermería practique lo suficiente para decirnos cómo hay que convertir sus teorías de la enfermería en enfermería práctica"20.
No ha sido fácil lograr una delimitación teórico-conceptual sobre el objeto de estudio que diferencia la medicina de la enfermería; es claro que cuando se trata de la atención a la salud del individuo, convergen varios profesionistas y que la línea que separa a uno de otro, a veces es muy delgada, sobre todo, entre estas dos profesiones. Una de las entrevistadas lo expresa de la siguiente manera:

[...] Intentar establecer una teoría propia, un lenguaje propio...es una tarea fenomenal, porque haber caminado de la mano con el saber médico, para mí es muy difícil ahorita separarme cuando me pongo a discernir, a ver cómo voy a elaborar un diagnóstico de enfermería, pues de inmediato me remito al diagnóstico médico y lo siento como un referente necesario...no sé por dónde lo vamos a enfrentar, ya hay algunas intentonas... es una tarea pero de las más delicadas que se ha propuesto la profesión y ahívamos apenas [...](E-10).

En la actualidad, existe consenso entre las enfermeras sobre la diferencia entre diagnosticar y tratar, asuntos que le competen al médico, y cuidar, en sentido amplio, holístico, ontológico y epistémico. Sin embargo, no ha sido sencillo, en la práctica, considerar que las enfermeras deben tener una preparación y, principalmente, una convicción sobre la importancia de definir con claridad el valor que el cuidado de enfermería agrega a los servicios de salud que ofrecen las instituciones o las organizaciones.

Se hace imprescindible, entonces, para poder actuar con discernimiento, con criterio profesional, una fuerte argumentación conceptual, trabajar sobre el objeto epistémico, profundizar en las tres dimensiones cuya comprensión es esencial para el estudio de la identidad en Enfermería: el ser, que se refiere al ethos, a su indagación filosófica, al humanismo; el saber, que tiene que ver con la claridad teórica y metodológica, con el conocer, y el hacer o quehacer, que se relaciona con el ejercicio profesional y que tiene su origen en la prestación de un servicio a la población.

Al decir del personal entrevistado, es necesario que antes de pensar en las diversas estrategias que pueden proponerse para fortalecer los saberes de las enfermeras mexicanas, quede suficientemente claro el rumbo a seguir, es decir, cuál es la prospectiva que se vislumbra en la creación de escenarios viables para la profesión:

[...]necesitamos primero ponernos de acuerdo qué queremos para México...si tú detectas y todas estamos partiendo de las necesidades, bueno...qué caminito vamos a seguir, pero...cómo voy a seguir un camino, si no me respalda un trabajo de investigación [...](E-9). 
Otra de las situaciones que manifiestan como base es la armonía con el equipo de salud y particularmente con los médicos:

[...] ]en muchas ocasiones no es estar en pleito con la profesión médica porque cada uno tiene su campo delimitado y que bueno, en ocasiones el hilo es tan delgado que bien puedes pasar hacia el otro lado de una forma, de ellos hacia nosotros, como nosotros hacia el área médica, pero siento que si un médico y el propio paciente te ve muy segura de tus conocimientos, y con una seguridad fundamentada, tanto en la práctica como en la teoría, yo creo que es ir ganando terreno como enfermeras, siempre he dicho que la enfermera debe ser una gente que mire a los ojos...que sea segura de su profesión, de sus conocimientos y de sus habilidades $[. .].(E-7)$.

Dentro de las principales alternativas que manifiestan las enfermeras entrevistadas para fortalecer los saberes de la profesión, se circunscriben en primer término a la preparación académica:

[...]aparte de la preparación...incluir aspectos filosóficos...los valores que la profesión debe tener y en qué consisten... dar la capacitación...transmitir ese conocimiento...la docencia como que ya lo tiene bien identificado, pero la asistencia no, se le sigue considerando...como que hacedora de procedimientos exclusivamente $[. .].(E-1)$.

Son las escuelas y facultades de Enfermería quienes deben iniciar con las innovaciones en sus planes de estudio:

[...]pudiéramos empezar por las escuelas y formando a los profesores [...] (E-5).

[...] Definir más su plan de estudios...profundizar un poco en qué perfil queremos que tenga nuestra egresada...para a su vez, poder elegir los contenidos del plan de estudios de enfermería [... ] (E-8).

También expresan que es muy importante la formación que va más allá de conocimientos técnicos, que deben tomarse en cuenta aspectos emocionales y psicológicos:

[...] Formación...tratar de simplificar, no simplificar como una acción de empobrecer, sino tratar de simplificar, para que la mayor parte de las enfermeras comprendan, y que con eso recuperen su autoestima, porque primero, para que tu puedas ser una buena enfermera tienes que tener un reconocimiento, no de la gente, sino de ti misma, de que eres importante en lo que haces socialmente, segundo, entender la naturaleza de enfermería y de la importancia del cuidar en una sociedad...al tener ya un reconocimiento de su campo...lo pueda proyectar hacia la sociedad, esto es vital, porque uno hasta que no siente orgullo de algo no lo presume, no lo muestra y el problema es que como no nos sentimos orgullosas pues no lo demostramos en forma personal...por eso tendríamos que entender qué es el cuidado [...] (E-6).

El empleo de la investigación como herramienta que permita dilucidar y fundamentar los saberes que guían la práctica es otra de las sugerencias:

[...]debería de ser lo de la creación...de evidencias cientificas de tu quehacer, nosotros no hemos podido hacer las guías por falta de evidencias, no hay evidencias en ningún lado, en internet no hay nada de enfermería...eso te está hablando de la investigación, de fortalecer la investigación en las instituciones de salud y hacerla conjuntamente con la docencia[...] (E-5).

[... ]empezarlo a construir a partir de generar grupos de trabajo...fundamentar nuestro trabajo...partir de la investigación como un elemento central, medular [...].(E-4).

Una estrategia que consideran decisiva es la integración docencia-asistencia, concebida como el conjunto de esfuerzos entre comunidad, universidad y servicio, encaminados a transformar las condiciones de salud de la población, representa una acción que permite un acercamiento a la realidad concreta donde se generan los problemas, la posibilidad de que surja y se forme personal más conciente y crítico de esa realidad, y que en una constante interacción y retroalimentación de ambas instancias pueda incidirse progresivamente en la transformación de las prácticas que se dan en los servicios:

[...]creo que la parte tanto asistencial como docente tiene que ir vinculada...mucho más integrada, que las facultades empiecen a trabajar con las enfermeras dentro de las instituciones $[\ldots](E-10)$. 


\section{CONSIDERACIONES FINALES}

Los modelos de desarrollo económico por los que ha transitado nuestro país, han delineado las políticas y tendencias en materia de salud y educación; lo que ha ido modificando diversas concepciones, tales como salud, enfermedad, universidad, educación, etc. La Universidad, por ejemplo, a mediados del siglo XX es vista como la institución que forma recursos humanos que favorecen el desarrollo industrial y de servicios; en la actualidad, se coloca al empleo como factor central del fin educativo. La formación integral del ser humano queda postergada y la educación se considera como una inversión ${ }^{3}$.

En el mismo sentido, la salud era conceptuada como la ausencia de enfermedad, su mantenimiento y preservación, en el Estado benefactor, se consideraba corresponsabilidad social; bajo el neoliberalismo, se especula la privatización del sector salud, fundamentalmente a la luz de cuatro argumentos: $\mathrm{El}$ servicio de atención en salud es muy caro para ser una actividad que pueda ser garantizada por el Estado; la atención en salud no es una cuestión pública, el Estado debe ocuparse de la prevención y la educación; se discute el proceso solidario del financiamiento por la vía de los impuestos o por los mecanismos de la seguridad social y; se liberaliza la demanda en la búsqueda de un paquete de atención, mediante acuerdos de los involucrados.

Los límites y alcances del cuidado de la salud por las enfermeras en un hospital público, en su mayoría, están encaminados al trabajo profesional técnico especializado, característica de los hospitales de este tipo, por el número de pacientes, enfermedades y complicaciones que estas presentan. Las enfermeras refieren dar cuidados específicos y generales tanto al paciente y al familiar que lo acompaña en su padecimiento, de ahí, que es necesario implementar un programa de cuidados de salud dirigidos a los familiares que cuidan al paciente, con el fin de prevenir alteraciones de salud en ellos, más aún, si el padecimiento es crónico degenerativo.

Aunque, la educación de las enfermeras se ha incrementado como resultado de las politicas educativas y de salud, el cuidado profesional no ha permeado en la atención del paciente, por la rutina y las politicas del sistema de salud. Además de que las enfermeras consideran que cuidar es realizar procedimientos aislados en los enfermos y no vislumbran los actos de cuidado para restablecer y mantener en equilibrio la vida. De ahíque la profesión de enfermería se encuentra en un proceso de transición y de consolidación que gira en torno a un sistema complejo como lo es el ser humano.

En los albores del siglo XXI, reconocer que el ser y hacer del personal de enfermería en México, se fundamenta en el paradigma vida-salud y no salud-enfermedad, es uno de los grandes desafíos. Lo es, porque como ya quedó señalado y comentado en el recuento histórico-sociológico que hemos hecho en este artículo, la profesión de enfermería, y el propio sistema de atención a la salud en México, sustenta un modelo de atención hospitalario, curativo y, aludiendo a los avances tecnológicos, mayormente invasivo, donde se sigue privilegiando la atención a la enfermedad (segmentada) del cuerpo. Todo ello hace difícil, lenta y compleja la transformación paradigmática del ser y hacer de enfermería, como profesión cuyo objeto epistémico de estudio es cuidar la vida y la salud de los seres humanos.

\section{Referências}

1.Jara NMI. Ética de la salud como ética de la vida. Gerencia y Políticas de la Salud 2000 nov; 1 (1): 86-102.

2.Nicolescu B. La transdisciplinariedad. [serial en línea] 2008 sep [citado mar 2000] [4 pantallas]. Disponible en: http://www.unav.es/ $\mathrm{cdb} /$ dhbapsalud.htmltitre.

3.Cárdenas BC. La profesionalización de la enfermería: Un análisis desde la sociología de las profesiones. México(MX): Pomares; 2005. 4.Nightingale F. Notas sobre enfermería: qué es y qué no es. Madrid(ES): Salvat; 1990.

5.Fuerbringer BM, Barrientos GE, et al. Poblanas en la salud pública de México. Historias de vida y semblanzas. Puebla: H. Ayuntamiento;2000.

6.Foucault M. Vigilar y castigar: nacimiento de la prisión. México(MX): Siglo XXI; 2000.

7.Foucault M. El nacimiento de la clínica. una arqueología de la mirada médica. México(MX): Siglo XXI; 1997.

8.Fuerbringer BM, Barrientos GE, et al. Enfermería disciplina científica: una aproximación epistemológica. México(MX): Universidad Autónoma de San Luis Potosí; 1995.

9.Secretaría de Educación Pública (MX). Dirección General de Profesiones. Comisión Técnica Consultiva de Enfermería, 9. México(MX): 2001.

10.Gaitán C, María C.La Enfermería en Colombia: orígenes de audacia y compromiso. Tunja, Boyacá(CO): Universidad Pedagógica y Tecnológica de Colombia; 1999

11.Colliere FM. Promover la vida.Madri (ES): Mc.Graw Hill;1993.

12. Holanda GA, Enedina S. Marcos conceituais na direção do cuidado: um estudo reflexivo do cuidado solidário de enfermagem. Esc Anna Nery Rev Enferm 2000 abr; (4): 73-82.

13. Medina JL. La pedagogía del cuidado: saberes y prácticas en la formación universitaria. Madrid (ES): Laestes; 1998.

14. Hernández CJ. Historia de la enfermería: un análisis histórico de los cuidados de enfermería. Madrid (ES): Interamericana; 1995.

15.Durán VMAM. Indagación filosófica y la práctica de la enfermería. Facultad de Enfermería/ Universidad Nacional de Colombia; 1998. p. 26-36.

16.Barroso RZ, Torres EJM. Fuentes teóricas de la enfermería profesional: su influencia en la atención al hombre como ser biosocial. Rev Cubana Salud Publica. 2001 ene; 27(1): 8-11.

17.Fernández FC. Enfermería fundamental. Barcelona(ES): Masson; 1995.

18.García CM , Martínez MML. Historia de la enfermería. Madrid (ES): Harcourt; 2001.

19.Martínez PC. Marco teórico conceptual para la elaboración de los posgrados en enfermería. México(MX): Universidad de Guanajuato; 1998.

20 - Pérez RMT. Holismo: un paradigma de enfermería para el cuidado. Enfermeras 2000 ene; (36): 13.

\section{Notas}

a F. Nightingale consideró que una atención eficaz y oportuna de Enfermería debería incluir aspectos básicos de la vida cotidiana, tales como ventilación y calefacción salubridad de las casas, alimentación, luz, limpieza y la administración y cuidado de los pequeños detalles que buscaban principalmente prevenir las enfermedades.

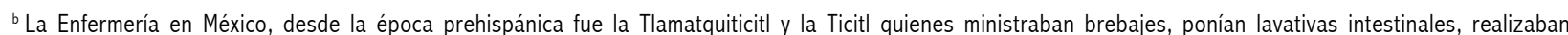
curaciones, daban fricciones, ponían férulas y vigilaban temascales, entre otras muchas actividades. A través del tiempo, mayoritariamente son las mujeres quienes han brindado cuidado a los enfermos, por tanto, se les ha considerado sanadoras "por naturaleza". 